\title{
Radiofrequency ablation of coronary sinus-dependent atrial flutter guided by fractionated mid-diastolic coronary sinus potentials
}

\author{
Joelci Tonet • Antonio De Sisti • Walid Amara • \\ Robert Frank • Françoise Hidden-Lucet
}

Received: 5 May 2010 / Accepted: 20 June 2010 / Published online: 4 September 2010

(C) The Author(s) 2010. This article is published with open access at Springerlink.com

\begin{abstract}
Background The efficacy of radiofrequency (RF) ablation of an uncommon coronary sinus (CS)-dependent atrial flutter (AFL) was evaluated using conventional electrophysiological criteria in a highly selected subset of patients with typical and atypical AFL.

Methods Fourteen patients with atrial flutter (11 males, mean age $69 \pm 9$ years) without previous right or left atrial $\mathrm{RF}$ ablation were included. Heart disease was present in eight patients. Baseline ECG suggested typical AFL in 12 patients and atypical AFL in two. Mean AFL cycle length was $324 \pm 64 \mathrm{~ms}$ at the time of RF ablation in the CS. Lateral right atrium activation was counterclockwise $(\mathrm{CCW})$ in 13 patients and clockwise in one. CS activation was $\mathrm{CCW}$ in all. Criteria for $\mathrm{CS}$ ablation included the presence of CS mid-diastolic fractionated atrial potentials (APs) associated with concealed entrainment with a postpacing interval within $20 \mathrm{~ms}$. Success
\end{abstract}

J. Tonet $\cdot$ R. Frank $\cdot$ F. Hidden-Lucet

Cardiology Institute, Rhythmology Unit,

Pitié-Salpêtrière Hospital,

Paris, France

A. De Sisti

Cardiology Department, Poissy-St Germain-en-Laye Hospital,

Poissy, France

\section{W. Amara}

Cardiology Department, Le-Raincy-Montfermeil Hospital,

Montfermeil, France

J. Tonet $(\square)$

Hôpital de La Pitié-Salpêtrière,

Institut de Cardiologie, Unité de Rythmologie,

47-83, Boulevard de l'Hôpital,

75651 Paris, France

e-mail: joelci.tonet@psl.aphp.fr was defined as termination of AFL and subsequent noninducibility.

Results The initial target for ablation was the cavotricuspid isthmus (CTI) in 11 patients and the CS with further CTI ablation in three. AP duration at the CS target site was $122 \pm 33 \mathrm{~ms}$, spanning $40 \pm 12 \%$ of the AFL cycle length. CS ablation site was located $1-4 \mathrm{~cm}$ from the CS ostium. Ablation was successful in all patients. Mean time to AFL termination during CS ablation was $39 \pm 52 \mathrm{~s}(<20 \mathrm{~s}$ in eight patients). No recurrence of ablated arrhythmia occurred during a follow-up of $18 \pm 8$ months.

Conclusions The CS musculature is a critical part of some AFL circuits in patients with typical and atypical AFL. AFL can be terminated in patients with CS or CTI/ CS AFL reentrant circuits by targeting CS mid-diastolic fragmented APs.

Keywords Atrial flutter. Coronary sinus .

Radiofrequency ablation

\section{Introduction}

There are few descriptions of macroreentrant atrial circuits in atypical atrial flutter (AFL) in patients who have not undergone previous atrial fibrillation (AF) radiofrequency (RF) ablation in the left atrium [1-5]. Ablation within the coronary sinus (CS) has occasionally been described in these patients $[6,7]$.

In patients with typical AFL, in whom cavotricuspid isthmus (CTI) ablation is the usual target site, unsuccessful procedures are uncommon [8]. RF ablation in the CS has never been described in these patients for AFL termination.

This study evaluated the efficacy of RF ablation inside the CS using conventional electrophysiological criteria in 
patients with typical or atypical AFL presenting with the $\mathrm{CS}$ as a critical part of the reentrant circuit.

\section{Methods}

\subsection{Study participants}

Inclusion involved 14 patients with 12-lead surface ECG AFL [9] in whom a mid-diastolic atrial potential (AP) inside the first centimeters of the CS was found to be part of the circuit after entrainment maneuvers. All patients underwent RF ablation in both the CTI and CS. Exclusion criteria included a previous history of ablation for $\mathrm{AF}$ in the left atrium; no patient had had previous ablation session at CTI. Written informed consent was obtained from all patients before enrollment in the study.

\subsection{Baseline clinical characteristics}

Between June 2005 and May 2010, 14 patients (11 males, mean age $69 \pm 8$ years) were included. These patients represented $<1 \%$ of all AFL ablations performed within the same time period.

The baseline clinical characteristics of the patients with AFL are summarized in Table 1. Eight patients had underlying structural heart disease (dilated cardiomyopathy in three, previous inferior myocardial infarction in one, aortocoronary bypass grafts in two, mitral valve repair in one, and mitral valve replacement in one). Seven patients had associated episodes of paroxysmal AF. Antiarrhythmic treatment failed to interrupt or prevent AFL (nine patients received amiodarone and one flecainide + amiodarone). Baseline 12-lead surface ECG morphology suggested typical AFL in 12 patients ("sawtooth" negative flutter waves in inferior leads, positive in V1) and atypical AFL in two. AFL was paroxysmal in two patients, persistent in six, and permanent in the remaining six.

\subsection{Electrophysiological study}

All patients were receiving oral anticoagulants before ablation. All procedures were carried out under mild sedation. Catheters were inserted via the right femoral vein. A steerable mapping catheter (10 poles, 2-8-2 mm electrode interspace) was positioned on the lateral wall of the right atrium in 12 patients, or along the tricuspid annulus (20 poles, 2-8-2 mm electrode interspace) in two; a quadripolar catheter (interspaced $5 \mathrm{~mm}$, MultiCath $4 \mathrm{M}$ catheter; Biotronik, Berlin, Germany) was positioned inside the CS. Ablation was carried out using a 4-mm-tip cooled RF catheter (IBI 1641; Saint Jude Medical, connected to a RF generator IBI-1500T8; Saint Jude Medical, St Paul, MN,
USA), or an 8-mm-tip RF catheter (Contact; Medtronic Inc., or Safire; Saint Jude Medical, connected to a RF generator Atakr II; Medtronic Inc., Minneapolis, MN, USA).

The CS ostium was located by observing the entry point of the quadripolar mapping catheter into the CS on left and right anterior oblique X-ray views using the ablation catheter in clockwise $(\mathrm{CW})$ rotation to define the $\mathrm{CS}$ ostium [6]. The location method was validated in the final patient included in this study (patient 14, Table 1) by retrograde CS angiography was carried out with a coronary artery catheter inserted into the CS (Fig. 1).

\subsection{Baseline electrophysiological characteristics}

The baseline electrophysiological characteristics of the patients with AFL are summarized in Table 1. Mean AFL cycle length before any RF attempt was $273 \pm 67 \mathrm{~ms}$. Intracardiac activation pattern at the right atrium (RA) lateral wall was counterclockwise (CCW) in 13 patients, and $\mathrm{CW}$ in one. The activation pattern in the $\mathrm{CS}$ was $\mathrm{CCW}$ in all patients.

Entrainment was performed $20 \mathrm{~ms}$ faster than the AFL cycle length. Sites with a postpacing interval (PPI) within $20 \mathrm{~ms}$ of the AFL cycle length were defined as being within the circuit. In the beginning of our experience, in three patients with a surface ECG morphology of typical AFL, and later in case no. 9, entrainment at the CTI was not carried out (to avoid AFL interruption or degeneration to AF). Entrainment at the CTI was then performed in 10 patients, of whom nine had a PPI within $20 \mathrm{~ms}$ of the baseline cycle length. Entrainment at CTI and CS before RF was made in five patients (cases 6, 7, 8, 13, 14), and in all of them, the PPI was within $20 \mathrm{~ms}$ at both sites.

In four patients (cases $6,7,8,14$ ), PPI was within $20 \mathrm{~ms}$ of the spontaneous cycle at the CTI (Fig. 2), proximal CS, and Bachman bundle zone, but $>50 \mathrm{~ms}$ in the mid- and distal CS. In three patients (cases $6,8,14$ ), PPI was $>50 \mathrm{~ms}$ at the mid-interatrial septum, while in case 7 , a nonexcitable electrically silent area was found at the interatrial septum. Pacing at different cycle lengths was not performed, either at CTI or CS. All entrainment maneuvers were made before RF attempts ablation.

\subsection{Radiofrequency ablation}

When initial RF ablation at the CTI failed to interrupt AFL, or when AFL with similar or different ECG surface morphology was still inducible despite the demonstration of a bidirectional CTI block [9], a subsequent attempt was conducted inside the CS. The criteria for targeting and ablating inside the CS were the presence of mid-diastolic 


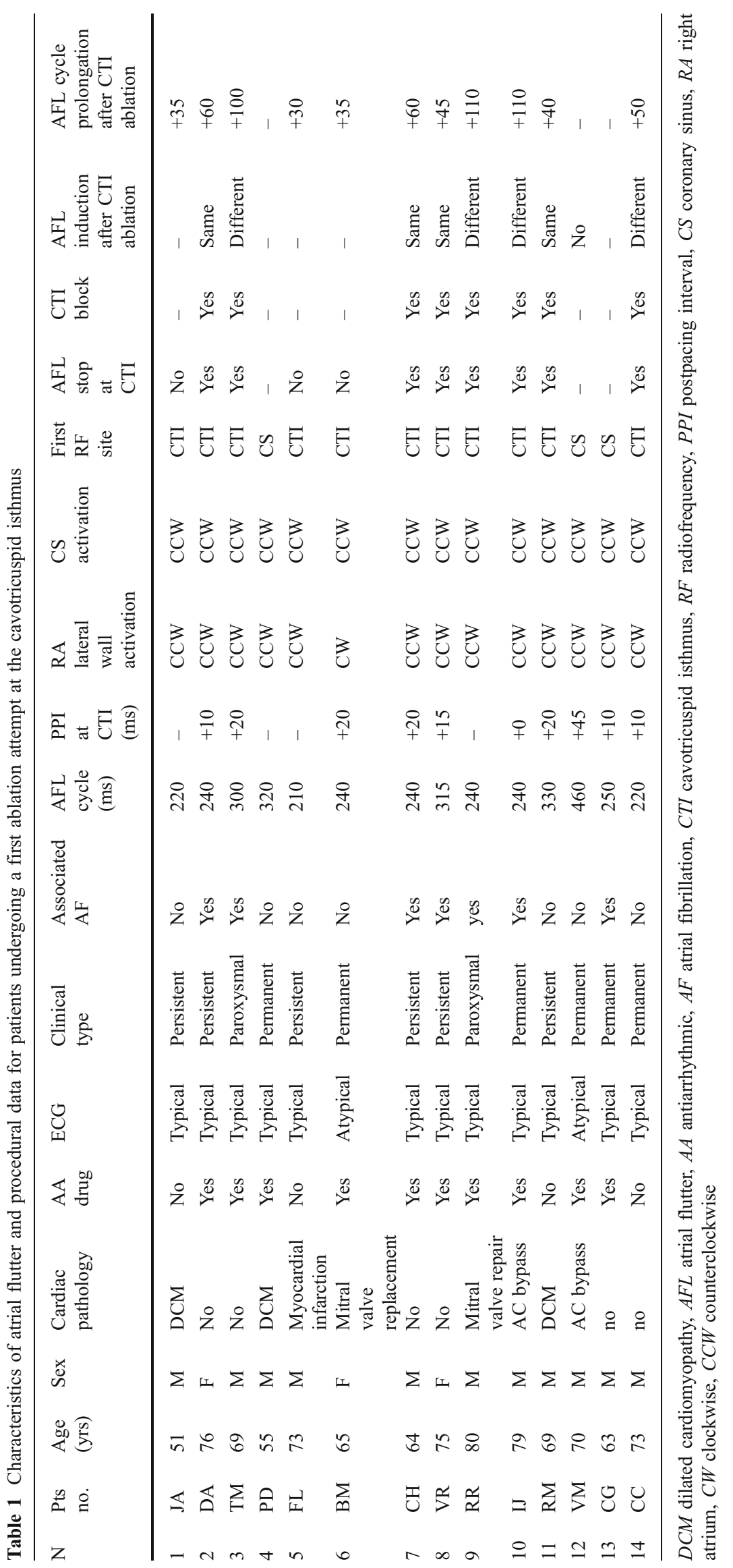




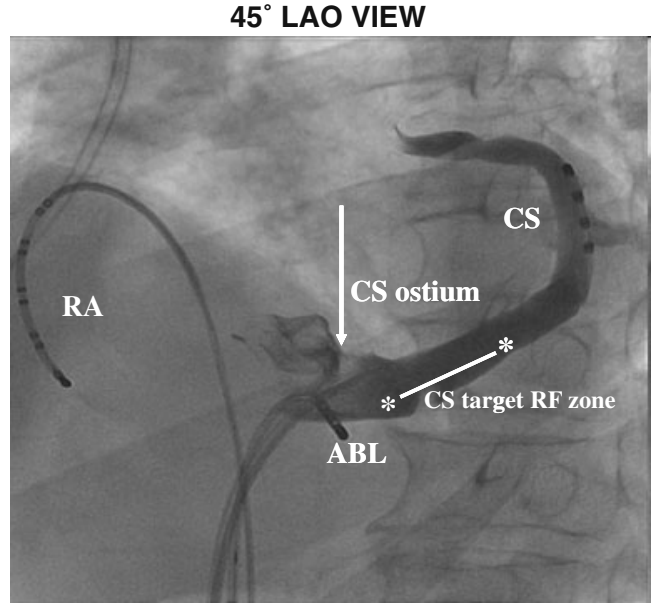

Fig. 1 Localization of the coronary sinus (CS) ostium by retrograde CS angiography (patient 14). Clockwise rotation of the ablation catheter defines the CS ostium in a $45^{\circ}$ left anterior oblique view. The CS target zone where radiofrequency ablation was applied $(1-4 \mathrm{~cm}$ from CS ostium) is shown between the two asterisks. $L A O$ left anterior oblique view, $A B L$ ablation catheter, $R A$ 10-pole catheter (interspaced $8-2-8 \mathrm{~mm}$ ) placed on the lateral wall of the right atrium, $C S$ coronary sinus catheter

coronary sinus fractionated prolonged APs (the upstroke of the surface F-wave, generally the V1 lead, was taken as the reference), associated with concealed entrainment showing a PPI within $20 \mathrm{~ms}$ [1, 6] (Figs. 3 and 4).

When RF ablation was delivered at the CTI, maximal power and temperature were $100 \mathrm{~W}$ and $60^{\circ} \mathrm{C}$ for the 8 -mm-tip catheter, and $40 \mathrm{~W}$ and $40-45^{\circ} \mathrm{C}$ for the 4-mm-tip cooled catheter. When ablation was carried out inside the CS, maximal power and temperature were $45 \mathrm{~W}$ and $55^{\circ} \mathrm{C}$ for the 8 -mm-tip catheter, and $25 \mathrm{~W}$ and $40^{\circ} \mathrm{C}$ for the 4-mm-tip cooled catheter.
In the case of early termination of AFL during RF delivery into the CS (within $20 \mathrm{~s}$ ), the RF pulse was maintained for $60 \mathrm{~s}$. When AFL was not rapidly terminated, the catheter was moved inside the CS around the zone of positive criteria.

$\mathrm{RF}$ ablation was not performed at septal isthmus position.

Acute success was defined as termination of AFL during RF delivery inside the CS, preceded by cycle-length prolongation and subsequent noninducibility. After arrhythmia termination, noninducibility was determined by programmed atrial stimulation, with up to three extrastimuli, or bursts up to $300 \mathrm{~ms}$ in cycle length. Induction tests are performed routinely after CTI ablation in our center to improve clinical outcome.

\subsection{Statistical analysis}

Values are presented as the mean \pm standard deviation or median, as appropriate.

\section{Results}

Results are summarized in Fig. 5.

The CTI was the first target for ablation in 11 patients. After ablation, AFL persisted in three patients, but the cycle length had increased without ECG, RA, and CS activation pattern modifications. In the other eight of 11 patients, CTI ablation interrupted AFL with a bidirectional CTI block [10], but AFL was still inducible in four patients with the same morphology and RA-CS activation pattern, but increased cycle length; in the other four, AFL was inducible, but the ECG morphology was different, atypical, and the cycle length was prolonged. Globally, in patients with persisting or
Fig. 2 Patient with surface ECG typical atrial flutter (AFL) (basic cycle length $250 \mathrm{~ms}$, patient 13). Concealed entrainment showed that both (a) the cavotricuspid isthmus and (b) the proximal coronary sinus (CS) were involved in the AFL circuit. This AFL was successfully interrupted by ablating into the CS. I, II, III, VI surface ECG leads, $A B L$ ablation catheter, $R A$ 10 -pole catheter placed at the lateral wall of the right atrium, $C S$ coronary sinus catheter
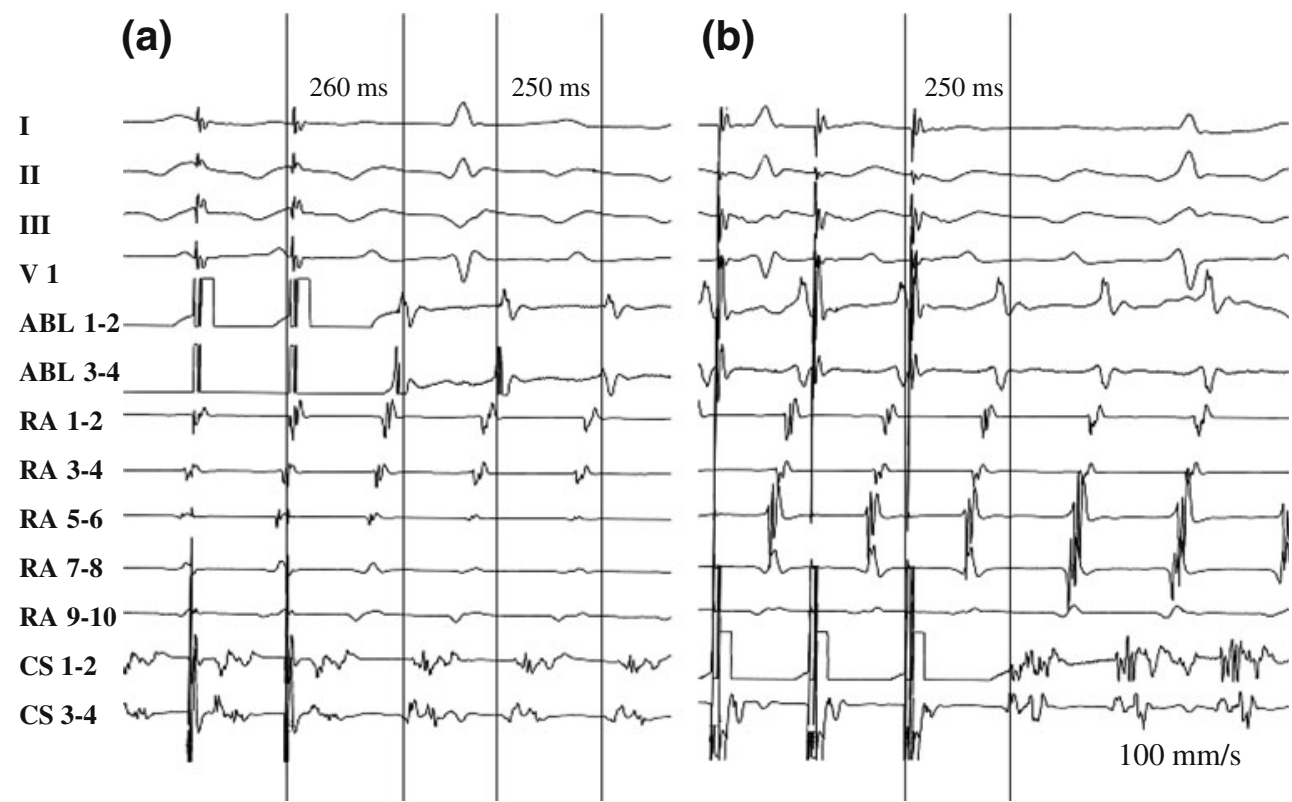
Fig. 3 Intracardiac recordings at the sites of successful ablation in the coronary sinus (CS). (a, b, c, and d) different types of mesodiastolic CS fragmented polyphasic potentials (the upstroke of the surface F-wave, generally V1 lead, was used as the reference). $I, I I, I I I, V 1$ surface ECG leads, $A B L$ ablation catheter (a)

I

V 1

II

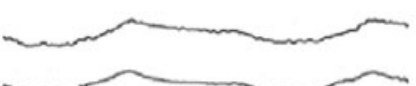

III

ABL 1-2

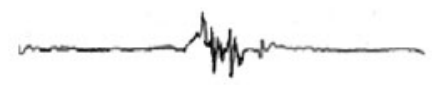

ABL 3-4

(b)

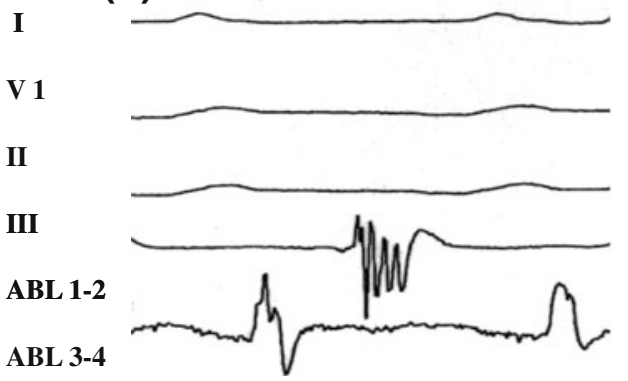

(c)

I

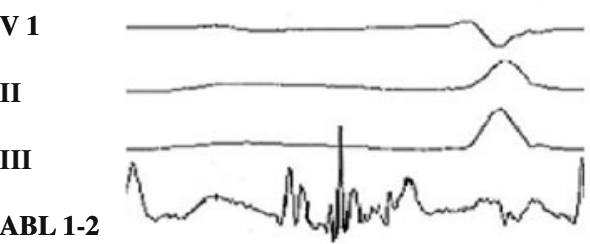

(d)

I

II

III

V 1

ABL 1-2

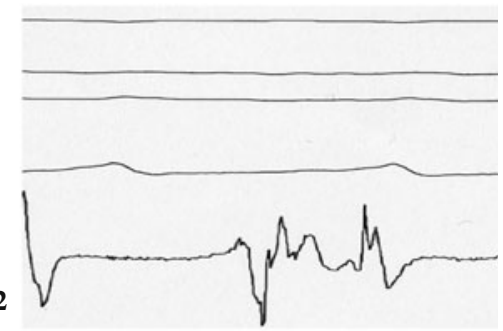

$200 \mathrm{~mm} / \mathrm{sec}$

reinduced AFL despite CTI ablation, the cycle length increased by $61 \pm 31 \mathrm{~ms}$.

In three patients, of whom two were among the last patients included (cases 12,13), the procedure began by directly ablating inside the CS. Of these patients, one had a long PPI at the CTI, and one had a PPI within $20 \mathrm{~ms}$ of the AFL basic cycle. AFL was interrupted in all three patients with subsequent AFL noninducibility.
3.1 Electrophysiological and procedural data during coronary sinus ablation

The electrophysiological and procedural data for the 14 patients during CS ablation are shown in Table 2.

At the time of RF ablation inside the CS, surface ECG morphology was typical in eight patients and atypical in six. Typical ECG morphology consisted of "sawtooth"
Fig. 4 Surface ECG typical atrial flutter (AFL) in patient one with basal cycle length $220 \mathrm{~ms}$ but CTI ablation was ineffective. Pacing was performed inside the coronary sinus $2 \mathrm{~cm}$ from the ostium where a mid-diastolic prolonged fragmented atrial potential was detected, and postpacing interval for entrainment matched the AFL cycle length. AFL was successfully terminated by delivering radiofrequency at this site. I, II, III, V1 surface ECG leads, $A B L$ ablation catheter, $R A$ 10 -pole catheter placed at the lateral wall of the right atrium, CS coronary sinus catheter

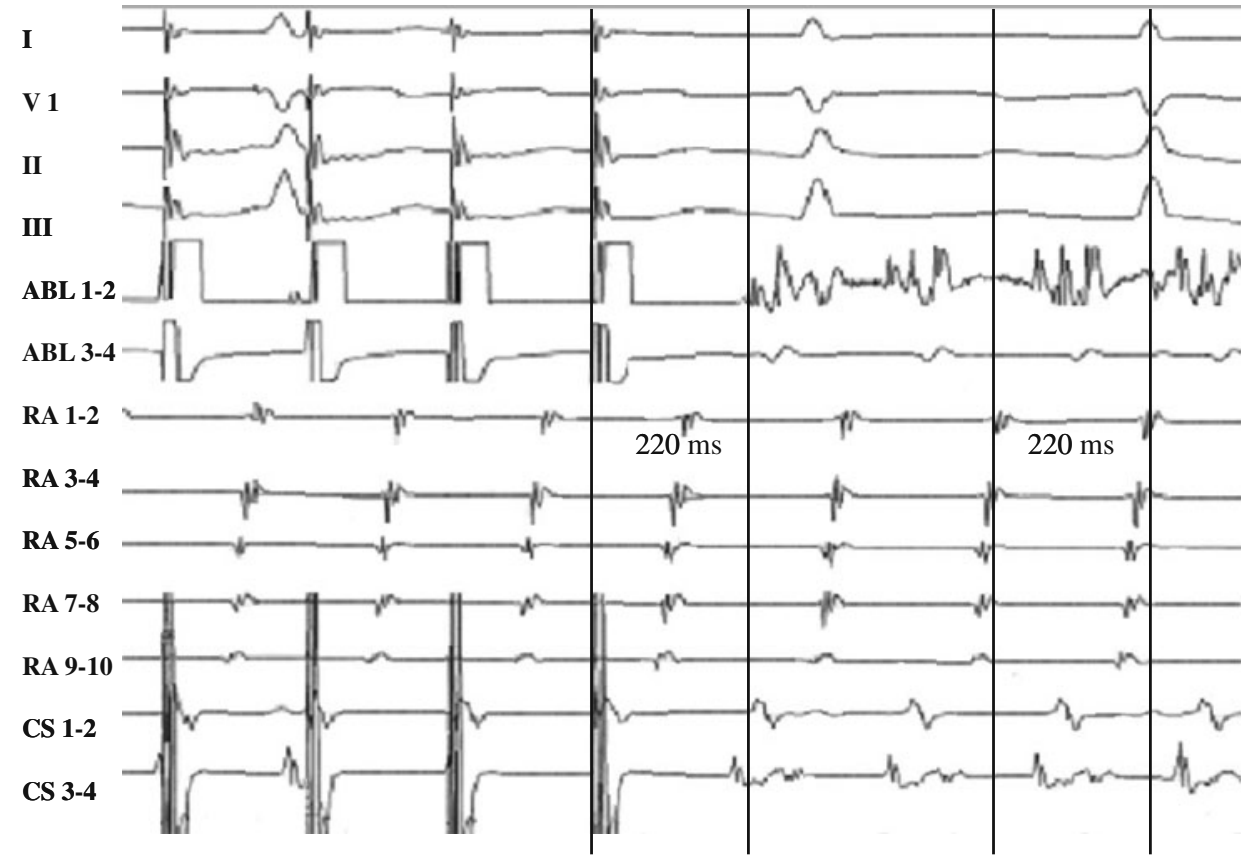

$100 \mathrm{~mm} / \mathrm{s}$ 
Fig. 5 Diagram illustrating the global results of radiofrequency ablation inside the coronary sinus

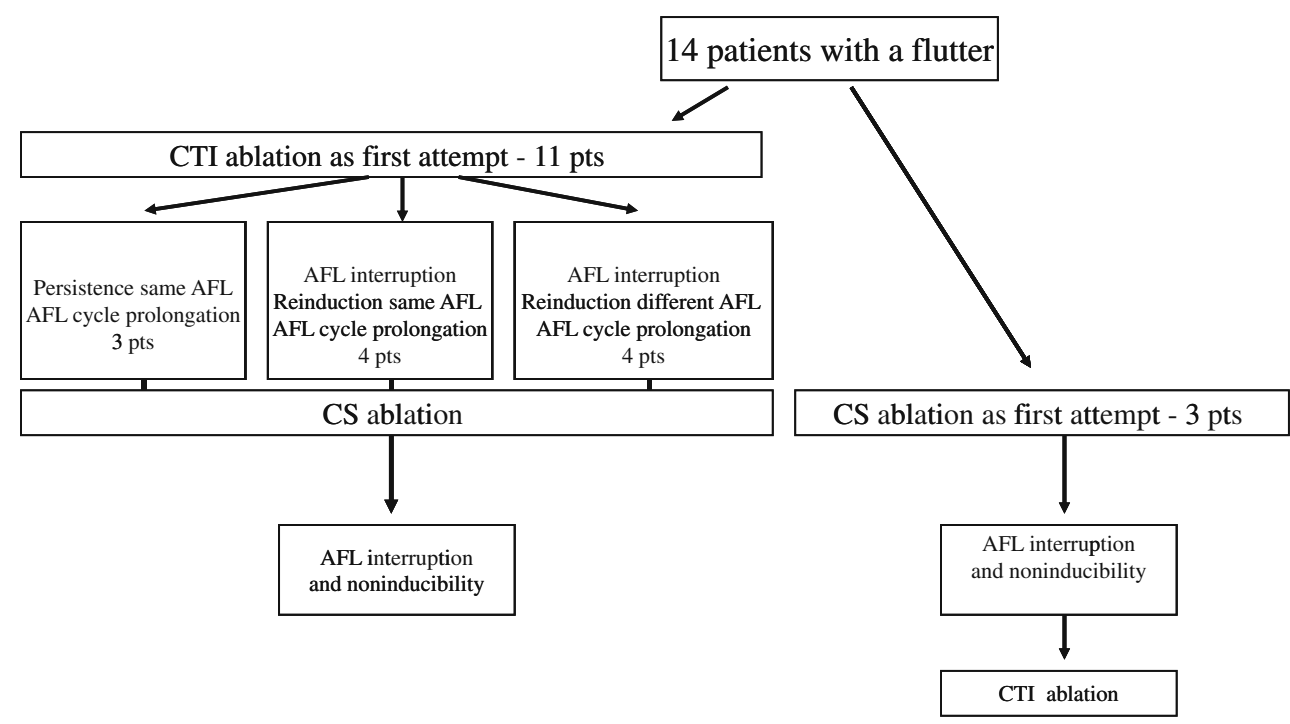

negative F-waves in the inferior leads and positive in V1. Atypical F-waves were characterized by low voltage, generally positive in inferior leads, often notched or biphasic, and generally positive in V1 [11].

Mean AFL cycle length was $324 \pm 64$ ms. The activation pattern at the RA lateral wall was $\mathrm{CCW}$ in 13 patients, and $\mathrm{CW}$ in one. The activation pattern in the CS was proximal to distal in all patients. AP duration at the targeted CS site was $122 \pm$ $33 \mathrm{~ms}$, spanning $40 \pm 12 \%$ of the AFL cycle length. Anatomically, the CS site to which RF was applied was located $1-4 \mathrm{~cm}$ from the CS ostium (median $2 \mathrm{~cm}$ ) [6] (Figs. 1 and 6).

RF ablation was performed using an 8-mm-tip catheter in nine patients and a 4-mm-tip cooled catheter in the remaining five. Rapid successful termination of AFL was achieved in all (11 with initial CTI ablation and three with initial ablation inside the $\mathrm{CS}$ ). The mean number of RF applications into the CS was $1.6 \pm 0.9$, and global RF time $88 \pm 50 \mathrm{~s}$. Time to AFL termination when ablating inside the CS (RF time from the beginning of RF delivery to AFL interruption) was $39 \pm 52 \mathrm{~s}(<20 \mathrm{~s}$ in eight patients; Fig. 7). Procedure and X-ray exposure durations were $68 \pm 23 \mathrm{~min}$ and $12 \pm 6 \mathrm{~min}$, respectively.

\section{Follow-up}

Data for hospital discharge and follow-up are summarized in Table 3.

All patients underwent regular visits to an outpatient clinic at 1-month and at subsequent 3-month intervals. Patients were maintained on anticoagulation therapy for at least 1 month after RF ablation. There was no interruption of antiarrhythmic treatment in the seven patients with a history of AF, while all antiarrhythmic drugs were withdrawn in the other seven.
During a mean follow-up of $18 \pm 8$ months, no patient had recurrence of the ablated arrhythmia, and only one patient had paroxysmal AF at 4-months follow-up.

\section{Discussion}

The CS may be a critical part of the reentrant circuit in patients with typical and atypical AFL. In some cases, both the CTI and CS are involved. These uncommon CS-dependent AFLs can be terminated by targeting mid-diastolic fragmented APs in the first few centimeters of the CS, with a PPI at this site suggesting the CS as an integral part of the circuit. Even if there is no atrial diastole in AFL, the concept of atrial coronary sinus mid-diastolic potential was a useful marker in approaching AFL-failed ablation. These CS-dependent AFLs may mimic typical CTI-dependent AFL [9] and cannot be predicted from ECG or clinical characteristics.

\subsection{Atypical AFL ablation}

In studies of atypical AFL with or without previous ablation for AF in the left atrium [1-5], RF ablation has mainly been performed by two approaches: linear lesions joining two anatomic barriers or silent areas, or both [1, 12] or, second, an electrophysiological approach targeting a critical isthmus presenting with a zone of slow conduction shown to be part of the circuit by pacing maneuvers [3, 4, 6]. Application of RF at this critical isthmus had been performed successfully with RF applications shorter than those usually needed for linear ablation [4, 6].

AFL termination by ablating inside the CS has been previously reported [6, 7]. An atypical AFL circuit involving the CS and left atrial myocardium was described by Olgin et al. [7], with circumferential successful ablation 


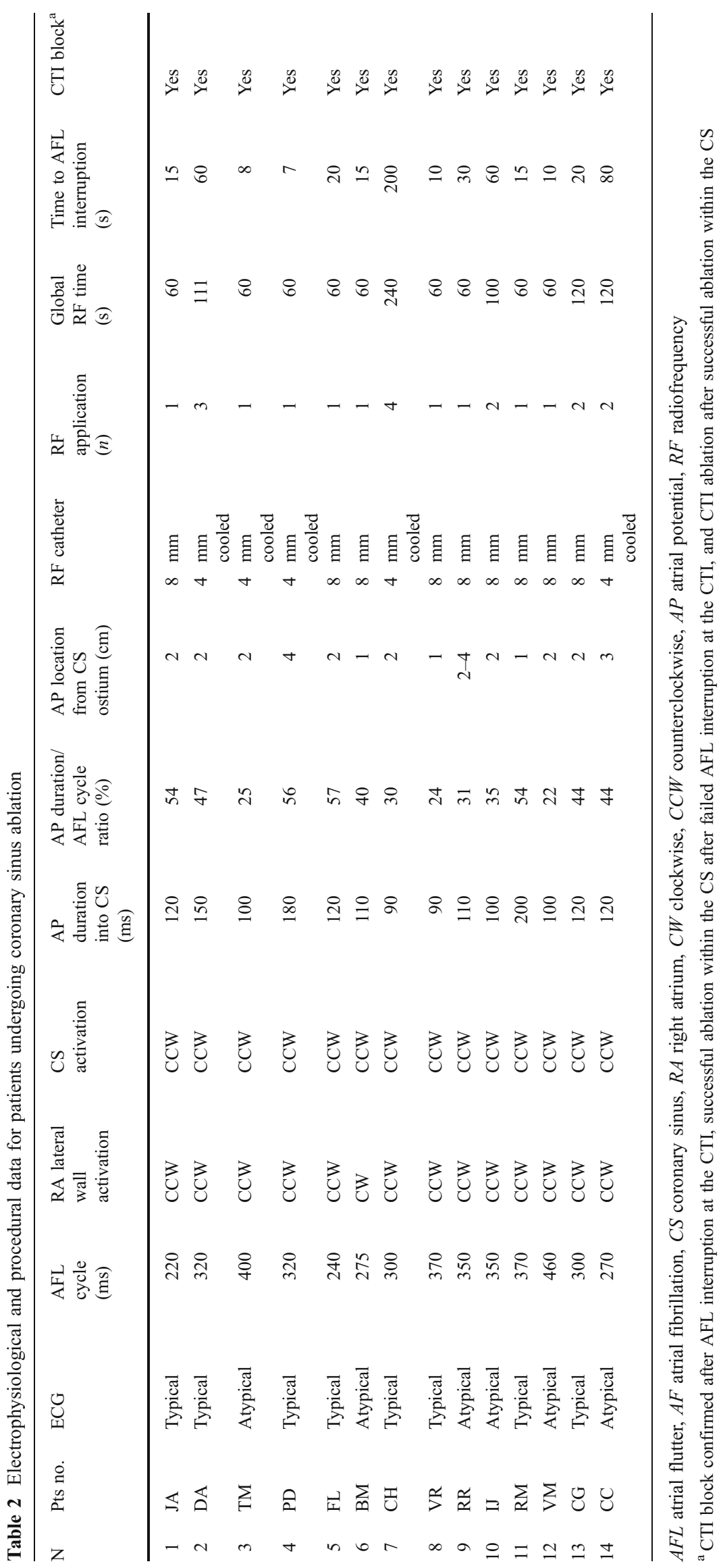


(a)

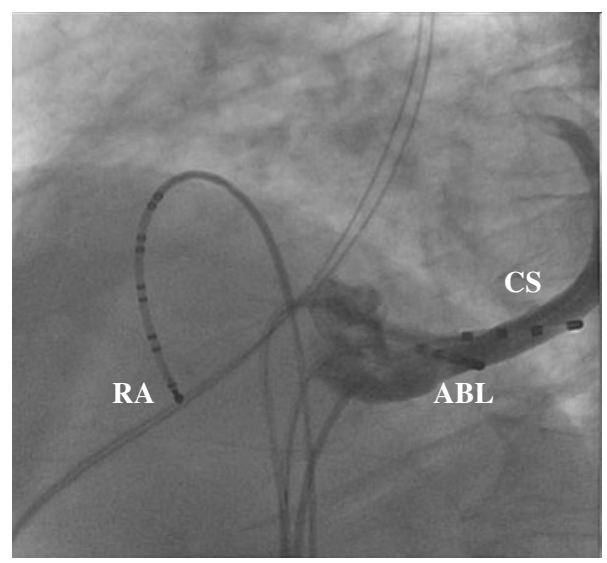

Fig. 6 Patient with typical atrial flutter (AFL) (cycle length $220 \mathrm{~ms}$, patient 14) successfully ablated at the cavotricuspid isthmus. A different atypical AFL with prolonged cycle length $(270 \mathrm{~ms})$ was subsequently induced. Ablation of this atypical AFL was successfully performed by targeting a CS mid-diastolic polyphasic atrial potential $3 \mathrm{~cm}$ from the ostium. No further arrhythmia was inducible.

within the distal CS. In the report of Della Bella et al. [6], two successful procedures were performed within the CS at a site where APs exhibited fragmented and prolonged middiastolic activity, and where concealed entrainment could be demonstrated.

To our knowledge, this is the first study to include a large number of patients with CS-dependent AFL who were ablated by targeting mid-diastolic APs inside the CS. The procedures were successful after a short RF application time. (b)

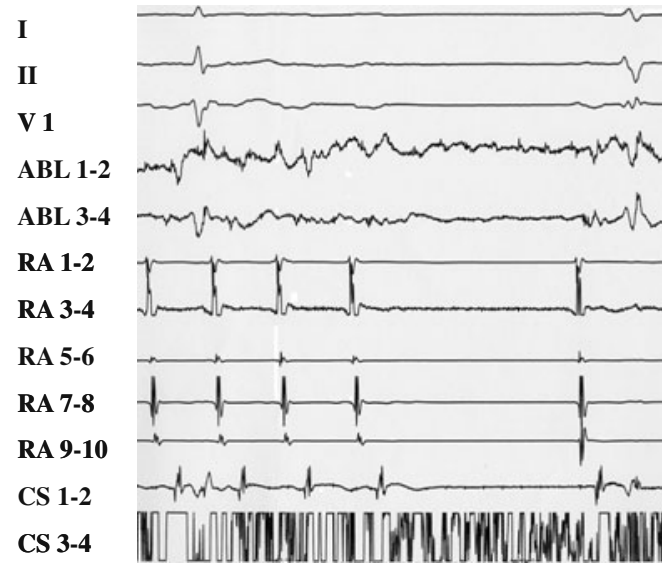

(a) Catheter location at the final successful site inside the CS confirmed by retrograde CS angiography. (b) Atypical AFL termination inside the CS. LAO left anterior oblique view, I, II, VI surface ECG leads, $A B L$ ablation catheter, $R A$ 10-pole catheter placed at the lateral wall of the right atrium, $C S$ coronary sinus catheter

\subsection{Coronary sinus anatomy and fractionated atrial potentials}

Anatomic studies [13-16] have shown that the proximal $4 \mathrm{~cm}$ of the CS is surrounded by a cuff of striated muscle, which is continuous with the RA myocardium at the CS ostium, and has multiple connections to the left atrial myocardium, and fewer connections to its distal portion. These muscular sleeves are extremely variable in number,
Fig. 7 Patient with typical surface ECG atrial flutter (AFL, patient 4). Ablation was first directly and successfully performed by targeting a coronary sinus mid-diastolic polyphasic potential $4 \mathrm{~cm}$ from the ostium; AFL rapidly terminated within $7 \mathrm{~s}$ of applying radiofrequency. Subsequently, no arrhythmia was inducible. The cavotricuspid isthmus was later successively ablated. $R F$ radiofrequency, $L A O$ left anterior oblique view, $I, I I, I I I$, $V 1$ surface ECG leads, $A B L$ ablation catheter, $R A$ 10-pole catheter placed at the lateral wall of the right atrium, $C S$ coronary sinus catheter

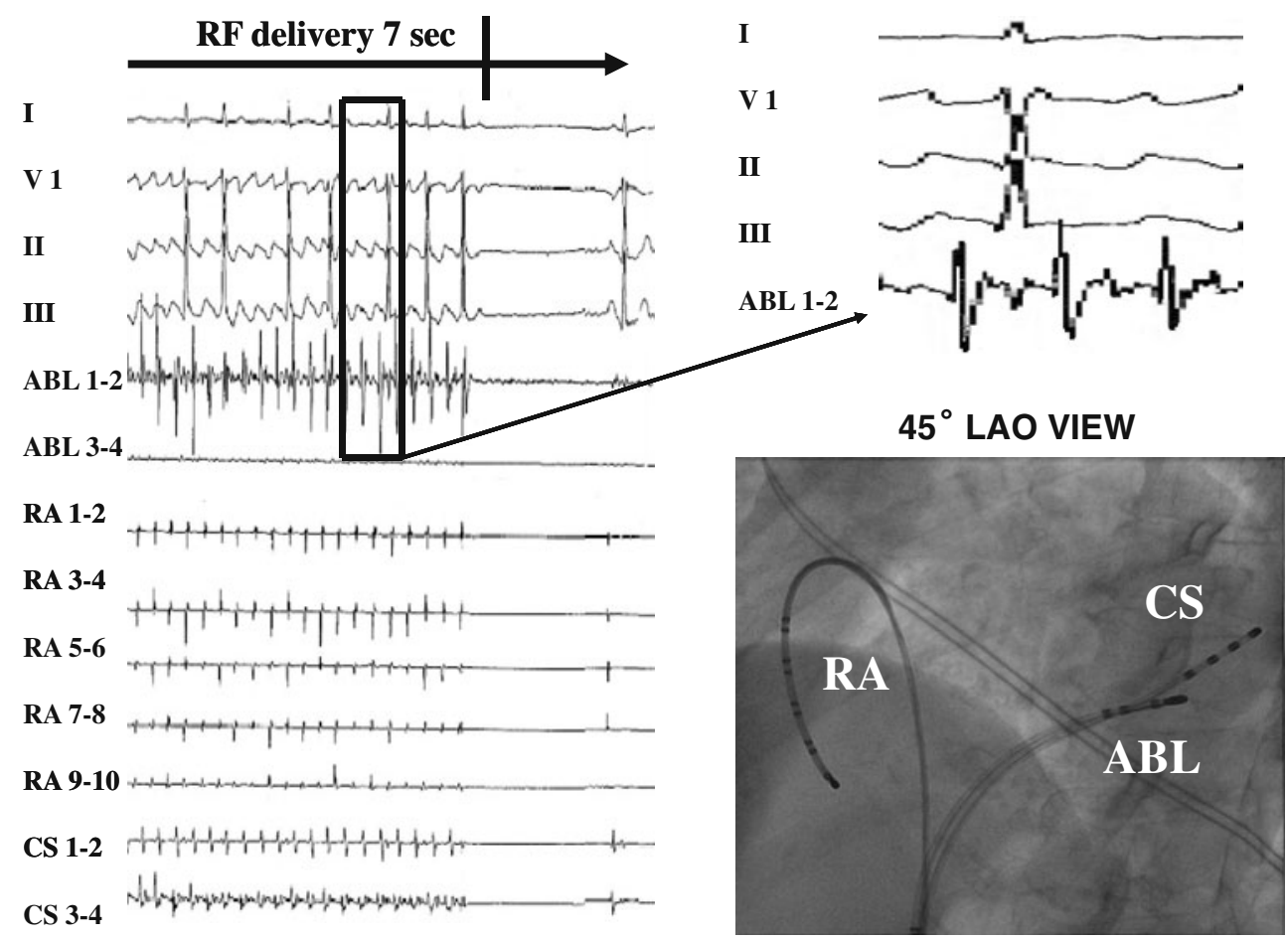


Table 3 Follow-up

\begin{tabular}{rllcl}
\hline N & Pts no. & AA drugs at hospital discharge & Follow-up (months) & Recurrence \\
\hline 1 & JA & No & 42 & SR \\
2 & DA & Amiodarone & 20 & SR \\
3 & TM & Amiodarone & 22 & SR \\
4 & PD & No & 21 & one episode of AF at 4 mo \\
5 & FL & No & 16 & SR \\
6 & BM & No & 16 & SR \\
7 & CH & Amiodarone & 14 & SR \\
8 & VR & Amiodarone & 25 & SR \\
9 & RR & Amiodarone & 15 & SR \\
10 & IJ & Amiodarone & 15 & SR \\
11 & RM & No & 12 & SR \\
12 & VM & No & 14 & SR \\
13 & CG & Amiodarone & 14 & SR \\
14 & CC & No & 9 & SR \\
\hline
\end{tabular}

$A A$ antiarrhythmic, $A F L$ atrial flutter, $A F$ atrial fibrillation, $S R$ sinus rhythm
Successful ablation was accomplished by circumferential RF application within the distal CS. Conversely, APs inside the CS in our patients were always fragmented and polyphasic (no spaced double potentials were recorded), and the successful CS target was more proximal.

Several reports $[19,20]$ described lower loop reentry as a variant of isthmus-dependent AFLs. In this case, the reentrant circuit turns around the inferior vena cava (IVC), or the IVC and tricuspid annulus in a figure-8-double loop reentry, both types involving also the CS musculature. Thus, CS involvement might suggest a similarity with our patients. Nevertheless, collision front in lateral RA described in lower loop reentry and RF interruption of these AFLs at ICT without needing of adjunctive RF lesions are not in agreement with our observations, making this mechanism improbable in our series. More recently, the same group [21] described in eight patients referred for typical AFL ablation, an intra-isthmus reentry circuit confined to the septal CTI, circling the CS ostium, and showing a collision wave front at the RA wall; in these AFLs, successful ablation required septal CTI RF applications. In our series, no patient presented with these electrophysiological characteristics, and no patient was ablated at the septal CTI.

\subsection{Surface ECG morphology and endocavitary activation pattern}

The correlation between AFL wave morphology and the underlying reentrant circuit is imperfect with infrequent but possible overlap between CTI and non CTI-dependent AFLs [22]. No typical and constant ECG pattern of CSdependent AFL was identified in this study. Several patients had a "sawtooth" ECG pattern in the inferior leads and positive $\mathrm{F}$ wave in $\mathrm{V} 1$, while others presented with variable ECG patterns. The intracardiac activation pattern in our potentials throughout the length of the CS were recorded. 
patients was also not useful for differentiating CSdependent AFL from CTI-dependent AFL. The ability to identify patients with CS-dependent AFL before ablation was not possible using surface ECG and endocavitary atrial activation patterns.

\subsection{Potential risk when ablating in the coronary sinus}

Delivery RF energy into the CS is often necessary to achieve complete block in the left mitral isthmus during a left atrial procedure for AF $[23,24]$. More recently, the CS has been a target for more extensive RF applications, including AF termination $[25,26]$. Ablation of the left atrial isthmus is often completed inside the CS on a line between the left inferior pulmonary vein and mitral annulus $[23,27]$. A case was recently reported of acute occlusion of the left circumflex coronary artery during left mitral isthmus linear ablation [28].

In our series, RF was applied in the first $4 \mathrm{~cm}$ of the CS, a zone where the wall is thick and arterial vessels are more distal. Additionally, the RF dose was generally low, contributing to the safety of ablation. In previous studies in humans and animals, ablation of epicardial located accessory pathways inside the CS was also shown to be relatively safe [29-33].

\subsection{Study limitations}

This study has several limitations. It included a heterogeneous study population with a complex rhythm disturbance with a lack of detailed mapping and standardization of ablation therapy. Mapping used conventional electrophysiological criteria, entrainment mapping was not systematically and extensively performed at sites other than the CS, and no electroanatomic map was planned. On the other hand, a pragmatic clinical approach was used, which was successful in terms of acute and long-term results.

\subsection{Clinical implications}

The results of this study strongly suggest that mid-diastolic APs in the CS should be evaluated systematically in patients with a "failed" CTI procedure for typical ECG AFL and in some patients with atypical AFL. This strategy may be help in avoiding an unnecessary transseptal approach for atrial left side ablation.

\section{Conclusions}

The CS musculature is a critical part of some AFL circuits in patients with typical or atypical AFL. In some cases, both the CTI and CS musculature seem involved in the reentrant circuit. These AFLs can be easily terminated by
CTI ablation combined with targeting CS mid-diastolic fragmented APs after demonstrating that the CS is an integral part of the circuit.

Open Access This article is distributed under the terms of the Creative Commons Attribution Noncommercial License which permits any noncommercial use, distribution, and reproduction in any medium, provided the original author(s) and source are credited.

\section{References}

1. Jaïs, P., Shah, D. C., Haïssaguerre, M., et al. (2000). Mapping an ablation of left atrial flutters. Circulation, 101, 2928-2934.

2. Kall, J. G., Rubenstein, D. S., Kopp, D. E., et al. (2000). Atypical atrial flutter originating in the right atrial free wall. Circulation, $101,270-279$.

3. Bogun, F., Bender, B., \& Hohnloser, S. H. (2000). Ablation of atypical atrial flutter guided by the use of concealed entrainment in patients without prior cardiac surgery. Journal of Cardiovascular Electrophysiology, 11, 136-145.

4. Ouyang, F., Ernst, S., Vogtmann, T., et al. (2002). Characterization of reentrant circuits in left atrial macroreentrant tachycardia: critical isthmus block can prevent atrial tachycardia recurrence. Circulation, 105, 1934-1942.

5. Fiala, M., Chovancík, J., Neuwirth, R., et al. (2007). Macroreentry tachycardia in patients without obvious structural heart disease or previous cardiac surgical or catheter intervention: characterization of arrhythmogenic substrates, reentry circuits, and results of catheter ablation. Journal of Cardiovascular Electrophysiology, 18, 824-832.

6. Della Bella, P., Fraticelli, A., Tondo, C., et al. (2002). Atypical atrial flutter: clinical features, electrophysiological characteristics and response to radiofrequency catheter ablation. Europace, 4, 241-253.

7. Olgin, J. E., Jayachandran, J. V., Engesstein, E., et al. (1998). Atrial macroreentry involving the myocardium of the coronary sinus: a unique mechanism for atypical flutter. Journal of Cardiovascular Electrophysiology, 9, 1094-1099.

8. Spector, P., Reynolds, M. R., Calkins, H., et al. (2009). Metaanalysis of ablation of atrial flutter and supraventricular tachycardias. The American Journal of Cardiology, 104, 671-677.

9. Saoudi, N., Cosio, F., Waldo, A., et al. (2001). A classification of atrial flutter end regular atrial tachycardia according to electrophysiological mechanisms and anatomical bases. European Heart Journal, 22(14), 1162-1182.

10. Saoudi, N., Ricard, P., Rinaldi, J. P., et al. (2005). Methods to determine bidirectional block of the cavotricuspid isthmus in radiofrequency ablation of typical atrial flutter. Journal of Cardiovascular Electrophysiology, 16, 801-803.

11. Bochoeyer, A., Yang, Y., Cheng, J., et al. (2003). Surface electrocardiographic characteristics of right and left atrial flutter. Circulation, 108, 60-66.

12. Marrouche, N. F., Natale, A., Wazni, O. M., et al. (2004). Left septal atrial flutter. Electrophysiology, anatomy, and results of ablation. Circulation, 109, 2440-2447.

13. Arruda, M., Widman, L., \& Antz, M. (1997). Myocardial continuity between right atrium and left atrium via the coronary sinus. Journal of the American College of Cardiology, 29, 358A.

14. Antz, M., Otomo, K., Arruda, M., et al. (1998). Electrical conduction between the right atrium and the left atrium via the musculature of the coronary sinus. Circulation, 98, 1790-1795. 
15. Chauvin, M., Shah, D. C., Haïssaguerre, M., et al. (2000). The anatomic basis of connections between the coronary sinus musculature and the left atrium in humans. Circulation, 101, $647-652$.

16. Kasai, A., Anselme, F., \& Saoudi, N. (2001). Myocardial connections between left atrial myocardium and coronary sinus musculature in man. Journal of Cardiovascular Electrophysiology, 12, 981-985.

17. Platonov, P. G., Mitrofanova, L., Ivanov, V., et al. (2008). Substrates for intra-atrial and interatrial conduction in the atrial septum: anatomical study on 84 human hearts. Heart Rhythm, 5, $1189-1195$.

18. Mitrofanova, L., Ivanov, V., \& Platonov, P. G. (2005). Anatomy of the inferior interatrial route in humans. Europace, 7(Suppl 2), 4955.

19. Yang, Y., Cheng, J., Bochoeyer, A., et al. (2001). Atypical right atrial flutter patterns. Circulation, 103, 3092-3098.

20. Zhang, S., Younis, G., Hariharan, R., et al. (2004). Lower loop reentry as a mechanism of clockwise right atrial flutter. Circulation, 109, 1630-1635.

21. Yang, Y., Varma, N., Keung, E. C., et al. (2005). Reentry within the cavotricuspid isthmus: an isthmus dependent circuit. Pacing and Clinical Electrophysiology, 28, 808-818.

22. Medi, C., \& Kalman, J. M. (2008). Prediction of the atrial flutter circuit location from the surface electrocardiogram. Europace, 10, 786-796.

23. Jaïs, P., Hocini, M., Hsu, L. F., et al. (2004). Technique and results of linear ablation at the mitral isthmus. Circulation, 110, 13511357.

24. Chugh, A., Oral, H., Good, E., et al. (2005). Catheter ablation of atypical atrial flutter and atrial tachycardia within the coronary sinus after left ablation for atrial fibrillation. Journal of the American College of Cardiology, 46, 83-91.
25. Haïssaguerre, M., Sanders, P., Hocini, M., et al. (2005). Catheter ablation of long-lasting persistent atrial fibrillation: critical structures for termination. Journal of Cardiovascular Electrophysiology, 16, 1125-1137.

26. Oral, H., Chugh, A., Good, E., et al. (2006). A tailored approach to catheter ablation of paroxysmal atrial fibrillation. Circulation, $113,1824-1831$.

27. Wittkampf, F. H., van Oosterhout, M. F., Loh, P., et al. (2005). Where to draw the mitral isthmus line in catheter ablation of atrial fibrillation: histological analysis. European Heart Journal, 26, 689-695.

28. Takahashi, Y., Jaïs, P., Hocini, M., et al. (2005). Acute occlusion of the left circumflex coronary artery during mitral isthmus linear ablation. Journal of Cardiovascular Electrophysiology, 16, 11041107.

29. Haïssaguerre, M., Gaita, F., Fischer, B., et al. (1992). Radiofrequency catheter ablation of left lateral accessory pathways via the coronary sinus. Circulation, 86, 1464-1468.

30. Sun, Y., Arruda, M., Otomo, K., et al. (2002). Coronary sinusventricular accessory connections producing posteroseptal and left posterior accessory pathways. Incidence and electrophysiological identification. Circulation, 106, 1362-1367.

31. Giorgberidze, I., Saksena, S., Krol, R. B., et al. (1995). Efficacy and safety of radiofrequency. Catheter ablation of left-sided accessory pathways through the coronary sinus. The American Journal of Cardiology, 76, 359-365.

32. Langberg, J., Griffin, J. C., Herre, J. M., et al. (1989). Catheter ablation of accessory pathways using radiofrequency energy in the canine coronary sinus. Journal of the American College of Cardiology, 13, 491-496.

33. Huang, S. K., Graham, A. R., Bharati, S., et al. (1988). Short- and long-term effects of transcatheter ablation of the coronary sinus by radiofrequency energy. Circulation, 78, 416-427. 